\title{
Asociación entre Presencia de Levaduras del Género Candida y Factores del Paciente Adulto Mayor con y sin Estomatitis Protésica
}

\author{
Association between Presence of Candida Yeasts and \\ Elderly Patient Factors with and without Denture Stomatitis
}

\author{
Ximena Lee M..; Leyla Gómez C."; Cristian Vergara N."; Elizabeth Astorga B." ; \\ Nataly Cajas C. \& Mariana Ivankovic S.
}

LEE, X.; GÓMEZ, C. L.; VERGARA, N. C.; ASTORGA, B. E.; CAJAS, C. N. \& IVANKOVOC, S. M. Asociación entre presencia de levaduras del género candida y factores del paciente adulto mayor con y sin estomatitis protésica. Int. J. Odontostomat., 7(2):279-285, 2013.

RESUMEN: La estomatitis protésica (ESP) es una alteración de la mucosa de soporte de diverso grado de severidad, que puede complicarse clínicamente con la presencia de levaduras del género Candida (LGC). Factores del individuo, del ambiente oral y del microorganismo favorecen la infección, que de no tratarse oportunamente puede evolucionar hacia lesiones hiperplásicas. Los objetivos fueron establecer relación clínico - microbiológica entre especies de LGC y ESP, asociar condiciones sistémicas (CS) prevalentes de pacientes portadores de prótesis con presencia y cantidad de LGC y efecto en la severidad de ESP; y asociar factores locales: uso continuo de prótesis y antigüedad protésica, con prevalencia de LGC y severidad de ESP. Un estudio observacional analítico fue realizado en 93 pacientes, edad promedio 67 años, $26 \%$ hombres y $74 \%$ mujeres, atendidos en el área de Prótesis Removible (Facultad de Odontología, Universidad de Chile), seleccionados de acuerdo a criterios de inclusión y firma del consentimiento informado. La recolección muestral de saliva no estimulada y mucosa oral se realizó considerando las variables del estudio. Estas muestras fueron procesadas de acuerdo a protocolos preestablecidos. El análisis microscópico permitió verificar desarrollo de colonias macromorfológicamente compatibles con LGC. Para el análisis estadístico se utilizaron test ANOVA y Kruskall Wallis. Existe relación clínica microbiológica entre presencia de LGC y ESP. El aumento de LGC se relaciona con severidad de ESP. La especie dominante es Candida albicans sobre no albicans. La presencia de ambas se asocia con aumento de la severidad de ESP. Al asociar las CS se observa asociación con depresión, no así con hipertensión arterial y diabetes mellitus. En relación a los factores locales protésico continuo y antigüedad protésica, existe relación entre antigüedad y presencia de LGC en pacientes con ESP, sin diferencias estadísticas entre presencia de LGC y uso protésico continuo en pacientes con ESP.

PALABRAS CLAVE: estomatitis protésica, levaduras, Candida.

\section{INTRODUCCIÓN}

La población chilena actual se caracteriza por tener un porcentaje importante de adultos mayores (AM), que según los datos aportados por la encuesta nacional de salud realizada en 2003 (MINSAL, 2003), un $69,8 \%$ es desdentado parcial y $29,10 \%$ total, $37,1 \%$ porta prótesis maxilares o mandibulares, $25,3 \%$ maxilar y $0,8 \%$ mandibular. Por otro lado, además del desdentamiento existen infecciones y lesiones de la mucosa oral de variada etiología, que en AM chilenos han sido poco estudiadas en relación a otras patologías. Un estudio descriptivo determinó que la prevalencia de una o más lesiones de la mucosa oral en AM de Santiago de Chile es $53 \%$, siendo la más común $(22,3 \%)$ la estomatitis protésica (ESP) (Espinoza et

\footnotetext{
Prof. Asistente. Académico Departamento de Prótesis, Facultad de Odontología, Universidad de Chile, Santiago, Chile.

** Prof. Asistente. Académico Departamento de Patología, Facultad de Odontología, Universidad de Chile, Santiago, Chile.

${ }^{* * *}$ Instructora. Académico Departamento de Prótesis, Facultad de Odontología, Universidad de Chile, Santiago, Chile.

***ticenciadas en Odontología. Facultad de Odontología, Universidad de Chile, Santiago, Chile.

Proyecto adscrito a PRI-ODO Año 2011 № 11 - 04 "Estudio cuantitativo de la ocurrencia de levaduras del género Candida en pacientes chilenos portadores y no portadores de prótesis, con o sin estomatitis protésica").
} 
al., 2003), que se define como un "proceso inflamatorio de la mucosa adyacente a la prótesis removible total o parcial" (Budtz-Jörgensen, 1974). Es una lesión generalmente asintomática, que afecta principalmente al paladar duro, y ha sido clasificada por Newton en tres tipos de acuerdo a severidad (Koeck, 2007): 1) Hiperemia puntiforme o tipo I (ESPI); 2) Hiperemia difusa atrófica o tipo II (ESPII, y 3) Hiperemia papilomatosa o tipo III (ESPIII). La etiología de ESP se asocia con factores de tipo (Emami et al., 2012; Koeck): 1) Mecánico, como consecuencia, por ejemplo, de desajuste o reaccional al uso protésico continuo. 2) Microbiano, producto de toxinas provenientes del biofilm o por proteasas de Candida albicans. 3) Endógeno tales como flujo salival disminuido, reducción de defensas inmunitarias o ciertas enfermedades o condiciones sistémicas. 4) Asociado a los materiales, como reacciones alérgicas hacia algún componente del acrílico. 5) Combinaciones de factores previos. 6) Origen desconocido. El tratamiento de la ESP, sugerido en "Guía clínica Salud Oral Integral para Adultos de 60 años" (MINSAL, 2010), es: 1. Controlar factores irritativos; 2 . Reforzar medidas de higiene oral y protésica. 3. Indicar antimicóticos tópicos y sistémicos frente a sospecha de infección fúngica.

En cuanto a las características de levaduras del género Candida (LGC), esta contiene más de 350 diferentes especies, pero sólo una proporción de ellas está implicada en enfermedades humanas (Williams et al., 2011). Están presentes en la flora normal de piel, mucosas y del aparato gastrointestinal, por lo cual el riesgo de infección endógena está siempre presente (Brooks et al., 2002). En general se acepta que el transporte comensal se presenta en aproximadamente el $50 \%$ de los individuos (Shimizu et al., 2008). Las LGC se encuentran en prótesis y mucosa oral de individuos con y sin signos de ESP. El papel de Candida, específicamente de $C$. albicans en el desarrollo de ESP, está asociado con crecimiento patógeno en superficies protésicas y de mucosa oral (Gendreau \& Loewy, 2011), siendo capaz de adherirse directamente o a través del biofilm al polimetilmetracrilato, lo cual favorece que la superficie protésica interna actúe como reservorio (Pereira-Cenci et al., 2008; Williams et al.). C. albicans es la principal especie patógena en ESP, aunque diversos estudios han aislado otras especies también involucradas en la patogénesis, como $C$. dubliniensis o C. glabrata (Salerno et al., 2011). El aparente aumento de especies no albicans, puede estar relacionado con métodos diagnósticos y técnicas moleculares más sensibles que permiten diferenciar especies y también por el aumento de resistencia ante agentes antifúngicos (Restrepo et al., 2003). Las LGC crecen en cultivo o tejidos como células ovales en gemación, de 3 a 6 u $m$ de tamaño. También forman pseudohifas cuando las yemas continúan su crecimiento pero sin desprenderse, generando cadenas de células alargadas, pinzadas o constreñidas en tabiques intercelulares. C. albicans es dimórfica, crecerá en forma unicelular o como hongo pluricelular dependiendo de las condiciones ambientales principalmente temperatura (Brooks et al.). Los métodos diagnósticos permiten confirmar la sospecha clínica de ESP asociada a LGC. Para la identificación de especies se utilizan pruebas de laboratorio basados en criterios macroscópicos, microscópicos, bioquímicos, inmunológicos, siembras en agar cromógenos y mediante técnicas de biología molecular.

Teniendo en cuenta entonces, que estudiar la ocurrencia de LGC en la cavidad oral de pacientes portadores de prótesis con y sin ESP, permitiría determinar qué cantidad de microorganismos podrían establecer la diferencia entre salud y enfermedad, y que cuantificar niveles altos de LGC en la cavidad oral podría indicar la necesidad de ejecutar acciones oportunas para prevenir o tratar la instalación de ESP, es que la presente investigación tuvo como objetivos: 1) Establecer relación clínico - microbiológica entre especies de LGC y ESP; 2) Asociar condiciones sistémicas prevalentes de pacientes portadores de prótesis con presencia y cantidad de LGC y efecto en la severidad de la ESP; 3) Asociar factores locales: uso nocturno de prótesis y antigüedad protésica, con prevalencia de LGC y severidad de ESP.

\section{MATERIAL Y MÉTODO}

La investigación corresponde a un estudio observacional analítico tipo caso-control (Hernández et al., 2006). La muestra estuvo conformada por 120 pacientes $\mathrm{AM}$ atendidos en el área de Prótesis Removible (Facultad de Odontología, Universidad de Chile) durante 2011, de los cuales 93 aceptaron voluntariamente participar y cumplían con los criterios de inclusión (Tabla I), edad promedio 67 años, $26 \%$ hombres y $74 \%$ mujeres. Los pacientes firmaron el consentimiento informado, aprobado por el Comité Ético Científico. Una vez definidas las variables del estudio (Tabla II), comenzó la etapa de recolección de muestras. En primer lugar, se realizó el examen clínico conformándose dos grupos de acuerdo a diagnóstico y categorizadas de la siguiente manera: 1) Pacientes 
desdentados totales o parciales, portadores de prótesis removible (PPR) sin ESP. 2) Pacientes desdentados totales o parciales, PPR con ESP. El diagnóstico clínico de ESP asociada a LGC se realizó según la clasificación de Newton. La categorización y el diagnóstico clínico fueron realizados por dos operadores calibrados supervisados por académicos responsables del estudio. Para la obtención de las muestras, a cada paciente se le solicitó: suspender el uso de colutorios 15 días antes de la recolección, 2 horas previas a la toma de las muestras debió estar en ayunas, no haber fumado ni realizado ningún procedimiento de higiene oral, y corroborar que no ha consumido antibióticos o antifúngicos por cualquier vía de administración. Estas indicaciones se les proporcionaron previamente por escrito. Para la toma de muestras de saliva no estimulada, cada individuo depositó en un tubo de ensayo estéril $2 \mathrm{ml}$ de saliva, el tubo fue sellado y rotulado. Las muestras fueron trasladadas en contenedor refrigerado al laboratorio de microbiología, siendo procesadas en un plazo inferior a $12 \mathrm{~h}$. Para la toma de muestras de la mucosa oral se frotó, con una tórula estéril humedecida con $100 \mu$ l de buffer fosfato de potasio $\mathrm{pH} 7.4$ estéril, la zona de la mucosa palatina. Las tórulas con muestras se introdujeron en tubos de ensayo estériles, y trasladadas refrigeradas al laboratorio para ser procesadas en un plazo inferior a $12 \mathrm{~h}$. El procesamiento de las muestras consistió en: para las muestras de saliva no estimulada, con el fin de obtener la cantidad de unidades formadoras de colonias (UFC) por ml de saliva, se realizó el método de recuento viable en placa de agar, diluyendo las muestras de saliva en buffer fosfato de potasio $\mathrm{pH}$ 7,4 estéril. Cada muestra de saliva fue agitada en un Vortex (Thermolyne Maxi Mix II) por 30 segundos para homogeneizarla; $100 \mu \mathrm{l}$ de saliva se diluyeron en $900 \mu \mathrm{l}$ de buffer fosfato de potasio $\mathrm{pH}$ 7,4 estéril, homogeneizándola en Vortex, de esta dilución se tomaron $100 \mu$ l diluidas nuevamente en $900 \mu$ de buffer fosfato de potasio $\mathrm{pH} 7,4$ estéril, que también se agitó en Vortex a velocidad máxima. Deambas diluciones $(1 / 10$ y $1 / 100)$ se sembraron $100 \mu$ l en placas de Agar Sabouraud suplementadas con Cloramfenicol $(0,5 \mathrm{mg} / \mathrm{ml})$.

Las muestras así procesadas fueron incubadas en estufa Pasteur a $37^{\circ} \mathrm{C}$ por 24 a $48 \mathrm{~h}$, en condiciones de aerobiosis. En cuanto al análisis microscópico, una vez finalizado el tiempo de incubación a las placas que presentaron menor desarrollo microbiano, se les realizó frotis y tinción de Gram a colonias macromorfológicamente compatibles con levaduras. Se observó con microscopio de transmisión (Zeiss, Axiostar plus) con aumento 100X, para confirmar que las colonias observadas en las placas están formadas por microorganismos micromorfológicamente compatibles con levaduras además de descartar la presencia de otros microorganismos. Para el recuento viable, se contabilizaron las colonias crecidas en la placa de agar, compatibles con LGC. El resultado de cada recuento se multiplicó por el factor de dilución obteniendo de esta forma las UFC de levaduras por $\mathrm{ml}$ de saliva. En relación a la muestra de torulado de mucosa bucal, esta fue sembrada con la misma tórula di-

Tabla I. Criterios de inclusión y exclusión.

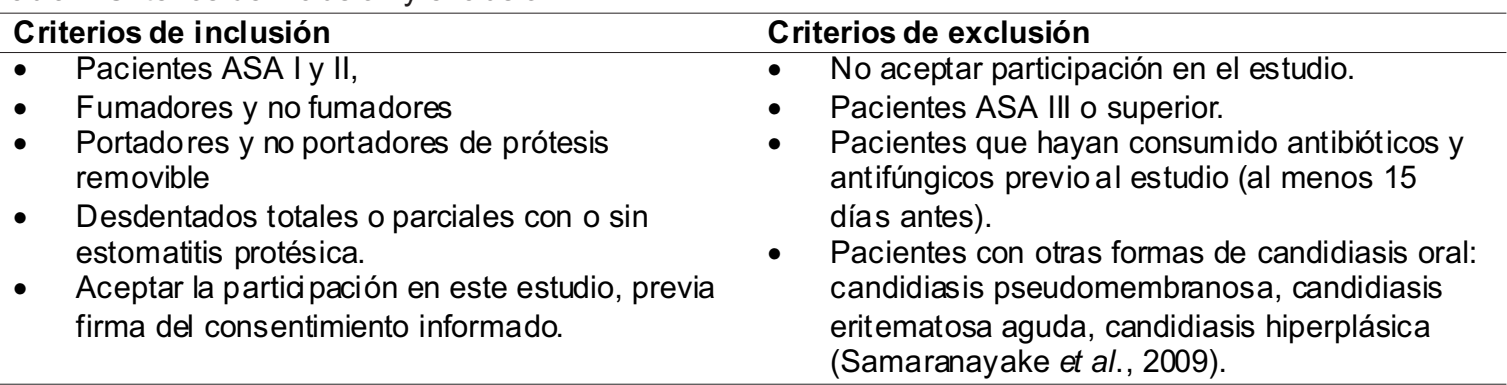

Tabla II. Variables del estudio.

\begin{tabular}{lll}
\hline Variables & Tipo de Variable & \\
\hline Estomatitis protésica & Ordinal & Sin ESP, ESPI, ESPII, ESPIII \\
Uso de prótesis (tiempo) & Nominal & Uso diurno, Uso protésico continuo \\
Antigüedad protésica & Cuantitativa & $0-4$ años, 5-9 años, +10 años \\
Recuento de LGC & Cuantitativa & UFC por ml de Saliva y torulado de mucosa oral \\
Identificación de LGC & Nominal & Candida albicans; Candida no albicans \\
\hline
\end{tabular}


rectamente, sin diluciones, en Agar Sabouraud suplementado con Cloramfenicol $(0,5 \mathrm{mg} / \mathrm{ml})$. Se incubó en estufa Pasteur a $37^{\circ} \mathrm{C}$ por 24 a $48 \mathrm{~h}$. Transcurrido el período de incubación se analizó la placa de agar para verificar presencia o ausencia de colonias. Para el análisis microscópico a las placas que presentaron desarrollo, se les realizó frotis y tinción de Gram a colonias macromorfológicamente compatibles con levaduras, se observó al microscopio con objetivo de inmersión (100X), para verificar que las colonias observadas están formadas por microorganismos micromorfológicamente compatibles con levaduras. Para el análisis macroscópico, se realizaron observaciones directas de las placas de agar para verificar desarrollo de colonias. Aquellas placas con desarrollo de colonias macromorfológicamente compatibles con LGC se consideraron positivas para prevalencia del microorganismo estudiado en mucosa oral. Finalmente, para el análisis estadístico se utilizaron los Test ANOVA y Kruskall Wallis.

\section{RESULTADOS}

Para establecer la relación clínico microbiológica entre especies de LGC y ESP, los recuentos obtenidos de LGC se ordenaron en intervalos de 0 a 1000, 1001 a 100.000, y sobre $100.001 \mathrm{UFC} / \mathrm{ml}$ de saliva, siendo bajo, mediano y alto recuento respectivamente. Los resultados fueron tabulados en la Tabla III y analizados estadísticamente. Según el test de Kruskall Wallis, existe diferencia estadística $(p=0.00)$ entre severidad de ESP y recuento de LGC. En relación a identificación de LGC y su relación con pacientes con o sin ESP, según severidad, los resultados develaron que $54 \%$ de ellos presentó LGC, $72 \%$ C albicans, $14 \%$ C. no albicans y $14 \%$ C. albicans y C. no albicans. Por lo tanto, C. albicans es la especie más prevalente. La identificación entre pacientes con y sin ESP fueron tabulados (Tabla IV) y analizados estadísticamente. El test ANOVA develó que la asociación entre severidad de ESP y presencia/especies de LGC, tiene diferencia estadística $(p=0,01)$.

Para asociar CS de pacientes portadores de prótesis con tipo de recuento en UFC/ml de LGC, se asociaron las siguientes variables: uso de prótesis, condición o enfermedad sistémica (CS): depresión (D), diabetes mellitus (DM) e hipertensión arterial (HTA); y tipo de recuento de LGC, bajo, mediano y alto: 0 a 1000, 1001 a 100.000 , y sobre $100.001 \mathrm{UFC} / \mathrm{ml}$ de saliva respectivamente. Cabe destacar que las tres CS seleccionadas, corresponden a aquellas más prevalentes en la población chilena. Los datos fueron tabulados (Tabla V), aplicándose el test estadístico ANOVA, que develó diferencia estadística para $D(p=0,0237)$, no así para DM $(p=0,7073)$ e HTA $(p=0,5702)$. Con respecto a la identificación de LGC de pacientes sin prótesis, y con prótesis y CS (D, DM, HTA), los datos obtenidos fueron consignados en la Tabla VI. Se aplicó el Test ANOVA que mostró diferencia estadística en CS D ( $p=0,0237)$; en relación a DM e HTA no se obtuvo diferencia estadística $(p=0,6430$ y 0,5702 respectivamente). En tanto a la asociación entre severidad de ESP y CS prevalente, los resultados se presentan en la Tabla VII. Los datos obtenidos fueron comparados mediante el test de Kruskall Wallis, develando que pacientes con HTA, $39 \%$ = ESPI, $48 \%=$ ESPII y $13 \%$ $=$ ESPIII, sin diferencia significativa $(p=0,98)$. Pacientes con DM: $37 \%=$ ESPI, $48 \%=$ ESPII y $15 \%=$ ESPIII, sin diferencia significativa $(p=0,72)$.

Tabla III. Relación entre cantidad, según tipo de recuento de LGC, con y sin ESP según severidad.

\begin{tabular}{lcccc}
\hline Tipo de recuento (UFC/ml) & Pacientes sin ESP (\%) & \multicolumn{3}{c}{ Pacientes con ESP (\%) } \\
\hline & $(39.7)$ & ESPI (24.7) & ESPII (25.8) & ESPIII (9.7) \\
Alto (>100.001) & 5.5 & 4.4 & 4.2 & 11.1 \\
Mediano (1001- 100.000) & 13.5 & 56.6 & 50.0 & 77.8 \\
Bajo (0-1000) & 81.0 & 39.0 & 45.8 & 11.1 \\
\hline
\end{tabular}

Tabla IV. Relación entre identificación de LGC y su relación con pacientes con y sin ESP, de diversa severidad.

\begin{tabular}{lcccc}
\hline Identificación LGC & Pacientes sin ESP (\%) 49.0 & \multicolumn{3}{c}{ Pacientes con ESP (\%) 51.0 } \\
\hline & & ESPI $=22.0$ & ESPII 25.0 & ESPIII =4.0 \\
Candida albicans & 24.0 & 67.0 & 39.0 & 50.0 \\
Candida no albicans & 0.0 & 0.0 & 15.0 & 0.0 \\
Candida albicans y no albicans & 8.0 & 8.0 & 7.0 & 50.0 \\
\hline
\end{tabular}


Por último, al asociar los factores locales: antigüedad protésica y uso protésico diurno o continuo con prevalencia de LGC y severidad de ESP, los resultados fueron los siguientes: 1) Para asociar antigüedad protésica y ausencia/ presencia de LGC, la muestra se dividió según años en 4 grupos: Grupo 1: 1 a 5; Grupo 2: 6 a10; Grupo 3: 11 a 20, y Grupo 4: >21. En la Tabla VIII, se agrupan los datos relacionando antigüedad protésica y presencia de LGC. Las variables se analizaron con test de Kruskal Wallis, develando diferencias significativas $(p<0,05)$. 2) Al asociar presencia de LGC y uso protésico continuo, los resultados in- dicaron que $83 \%$ de los pacientes que presentaron uso diurno hubo presencia de LGC. Aquellos que presentaron uso protésico continuo, la presencia de LGC fue $72 \%$, no siendo esta diferencia estadísticamente significativa $(p<0,05)$. Por lo tanto, en este estudio no hubo diferencia estadística entre presencia de LGC y uso protésico diurno o continuo en pacientes con ESP. Por otro lado, al asociar severidad de ESP y uso protésico continuo (Tabla IX), los datos obtenidos fueron comparados mediante test de Kruskall Wallis, develando que no existen diferencias estadísticamente significativas en este grupo de pacientes $(p<0,05)$.

Tabla V. Relación entre tipo de recuento y su relación con pacientes con y sin prótesis, y CS asociada.

\begin{tabular}{lcccccc}
\hline Tipo de recuento (UFC/mI) & \multicolumn{3}{c}{ Pacientes sin prótesis (\%) } & \multicolumn{3}{c}{ Pacientes con prótesis (\%) } \\
\hline & D & DM & HTA & D & DM & HTA \\
Bajo (0-1000) & 74.0 & 69.0 & 75.0 & 65.2 & 71.4 & 62.0 \\
Mediano (1001- 100.000) & 24.0 & 24.0 & 17.9 & 25.5 & 28.6 & 33.2 \\
Alto (>100.001) & 2.0 & 7.0 & 7.1 & 9.3 & 0.0 & 4.2 \\
\hline
\end{tabular}

Tabla VI. Relación entre presencia de LGC y su relación con pacientes con y sin CS.

\begin{tabular}{lcccc}
\hline Identificación LGC & Pacientes sin prótesis (\%) & \multicolumn{3}{c}{ Pacientes con prótesis (\%) } \\
\hline & & D & DM & HTA \\
Ausencia & 49 & 5.9 & 42.9 & 41.7 \\
Candida albicans & 37 & 64.7 & 57.1 & 41.7 \\
Candida no albicans & 8.3 & 23.5 & 0.0 & 8.3 \\
Candida albicans y no albicans & 5.1 & 5.9 & 0.0 & 8.3 \\
\hline
\end{tabular}

Tabla VII. Relación entre severidad de ESP y su relación con pacientes con CS

\begin{tabular}{lcc}
\hline Severidad ESP & \multicolumn{2}{c}{$\begin{array}{c}\text { Pacientes con CS } \\
\text { (\%) }\end{array}$} \\
\hline & \multicolumn{2}{c}{ HTA } \\
Tipo I & 37.0 & 39.0 \\
Tipo II & 48.0 & 48.0 \\
Tipo III & 15.0 & 13.0 \\
\hline
\end{tabular}

Tabla VIII. Relación entre antigüedad protésica y su relación con presencia de LGC

\begin{tabular}{lcc}
\hline Severidad ESP & \multicolumn{2}{c}{ Pacientes con CS (\%) } \\
\hline & DM & HTA \\
Tipo I & 37.0 & 39.0 \\
Tipo II & 48.0 & 48.0 \\
Tipo III & 15.0 & 13.0 \\
\hline
\end{tabular}

Tabla IX. Relación entre severidad de ESP y su relación con uso protésico continuo.

\begin{tabular}{lcc}
\hline Severidad ESP & \multicolumn{2}{c}{ Uso protésico continuo (\%) } \\
\hline & Sí & No \\
ESPI & 50.0 & 21.0 \\
ESPII & 33.0 & 58.0 \\
ESPIII & 17.0 & 21.0 \\
\hline
\end{tabular}

\section{DISCUSIÓN}

Al establecer relación clínico - microbiológica entre especies de LGC y ESP, este estudio ha develado que existe relación entre ambas variables. La cantidad de LGC es mayor en pacientes con ESP, y aumenta con la severidad según la clasificación de Newton. Llama la atención que $5,4 \%$ de pacientes sin ESP presentó alto recuento de LGC. En ESPII disminuye el mediano recuento respecto a ESPI, sin embargo, esta disminución se compensa con la expresión de alto recuento de LGC. Se concluye que existe relación entre presencia de LGC y ESP; $C$. albicans predomina sobre C. no albicans. La presencia de $C$. albicans y C. no albicans simultáneamente en cavidad oral, se relaciona con severidad de ESP en el grupo estudiado.

Al asociar diversas CS de pacientes portadores de prótesis con presencia y cantidad de LGC y efecto en la severidad de ESP, en este estudio, no se de- 
terminó mayor severidad de ESP en pacientes con CS. Se observó asociación entre presencia y cantidad de LGC y CS D, no así con DM e HTA, en las cuales no hubo diferencia estadística. Por lo tanto la $\mathrm{D}$ en PPR se asocia con mayor recuento de colonias, siendo C. albicans la especie más prevalente, pero con un porcentaje no despreciable de $\mathrm{C}$ no albicans. Por otro lado, es interesante que si bien es cierto en este estudio no fue estadísticamente significativa la asociación entre severidad de ESP y CS, no hay que perder de vista que las LGC son muy prevalentes y que por lo tanto los pacientes que presenten $D$ son más vulnerables frente a una invasión y potencial infección.

En relación a los factores locales uso protésico continuo y antigüedad protésica y su relación con prevalencia de LGC y severidad de ESP, en este estudio no hubo diferencia estadística entre presencia de LGC y uso protésico continuo en pacientes con ESP. Como conclusión, no se determinó una mayor severidad en la ESP de pacientes con uso protésico continuo al compararlo con pacientes con uso protésico diurno.
En relación al factor antigüedad protésica, se constató que existe relación entre tiempo de uso de prótesis removible en años y prevalencia de LGC. Se aprecia que la presencia de LGC aumenta con el tiempo de uso, con un incremento importante entre 6 a 10 años. Es interesante constatar también que posterior a los 10 años esta presencia de LGC disminuye, probablemente asociados a cambios en el ecosistema oral que sería importante estudiar.

Por lo tanto, establecer la ocurrencia de LGC en la cavidad oral con y sin ESP, nos permite aproximarnos a determinar qué cantidad de microorganismos podrían establecer la diferencia entre salud y enfermedad en un paciente AM portador de prótesis removible, específicamente en relación a severidad de ESP. Además, la cuantificación de niveles altos de LGC en la cavidad oral de los pacientes, podría indicar la necesidad de ejecutar acciones terapéuticas oportunas con el fin de prevenir o tratar la instalación de ESP de diversa severidad, como por ejemplo recambio protésico antes de 5 años y evitar el uso continuo de prótesis durante el día.

LEE, X.; GÓMEZ, C. L.; VERGARA, N. C.; ASTORGA, B. E.; CAJAS, C. N. \& IVANKOVOC, S. M. Association between presence of Candida yeasts and elderly patient factors with and without denture stomatitis. Int. J. Odontostomat., 7(2):279$285,2013$.

ABSTRACT: Denture stomatitis (DS) is a change of the support mucous of diverse severity that can get clinically complex with the presence of yeasts of the Candida genus (YCG). Individual's factors of the oral and microorganism environment favor the infection, which can evolve to hyperplasic lesions if not treated early. The aims were to establish clinical-microbiological relationship between YCG and DS species, associate systematic conditions (SC) prevalent of patients using prosthesis with presence and quantity of YCG and effect on DS severity; and associate local factors: continuous use of prosthesis and age of the prosthesis (AP), with YCG prevalence and DS severity. An analytical observational study was performed in 93 patients, average age 67 years old, $26 \%$ male and $74 \%$ female, treated at the Removable Prosthesis Area (Facultad de Odontología, Universidad de Chile), selected according to inclusion criteria and signature of informed consent. The unstimulated saliva and oral mucous sample recollection was performed considering the study's variables. The microscopic analysis allowed to check macro morphologically YCG compatible colonies development. For the statistical analysis, ANOVA and Kruskall Wallis tests were used. There is a microbiological clinical relationship between the presence of YCG and DS. The increase of YCG is related to the severity of DS. The dominant species is Candida albicans over not albicans. The presence of both is associated with the increase of DS severity. By associating the SC, a relation with depression is observed, but not with arterial hypertension and mellitus diabetes. As for the continuous local prosthetic factors and AP, a relationship exists between AP and presence of YCG in patients with DS, without statistical differences between YCG and continuous prosthetic use in patients with DS.

KEY WORDS: denture, stomatitis, Candida.

\section{REFERENCIAS BIBLIOGRÁFICAS}

Brooks, G.; Butel, J. \& Morse S., Microbiología médica. 17ª ed. México D. F.-Santa Fé de Bogotá, El Manual Moderno, 2002. pp.661-96. Capítulo 5.
Budtz-Jörgensen, E. The significance of Candida albicans in denture stomatitis. Scand. J. Dent. Res., 82(2):15190, 1974. 
LEE, X.; GÓMEZ, C. L.; VERGARA, N. C.; ASTORGA, B. E.; CAJAS, C. N. \& IVANKOVOC, S. M. Asociación entre presencia de levaduras del género candida y factores del paciente adulto mayor con y sin estomatitis protésica. Int. J. Odontostomat., 7(2):279-285, 2013.

Emami, E.; Taraf, H.; de Grandmont, P.; Gauthier, G.; de Koninck, L.; Lamarche, C.; et al. The association of denture stomatitis and partial removable dental prostheses: A systematic review. Int. J. Prosthodont., 25(2):113-9, 2012.

Espinoza, I.; Rojas, R.; Aranda, W. \& Gamonal, J. Prevalence of oral mucosal lesions in elderly people in Santiago Chile. J. Oral Pathol. Med., 32(10):571-5, 2003.

Gendreau, L. \& Loewy, Z. G. Epidemiology and etiology of denture stomatitis. J. Prosthodont., 20(4): 251-60, 2011.

Hernández, R.; Fernández, C. \& Baptista, P. Metodología de la Investigación. $4^{a}$ ed. México D. F., McGraw-Hill Interamericana, 2006.

Koeck, B. Prótesis Completas. $4^{\mathrm{a}}$ ed. München, Urban \& Fischer, 2007. pp.344-6. Cap. 13.

MINSAL. Guía Clínica Salud Oral Integral para Adultos de 60 años. Santiago, Ministerio de Salud, 2010. Disponible en: http://www.redsalud.gov.cl/archivos/guiasges/ saludoral60.pdf

MINSAL. Encuesta Nacional de Salud. 2003. Disponible en: http://epi.minsal.cl/epi/html/invest/ENS/ENS.html.

Pereira-Cenci, T.; Del Bel Cury, A. A.; Crielaard, W. \& Ten Cate, J. M. Development of Candida-associated denture stomatitis: new insights. J. Appl. Oral Sci., 16(2):86-94, 2008.

Restrepo, A.; Robledo, J.; Leiderman, E.; Restrepo, M.; Botero, D. \& Bedoya, V. Enfermedades infecciosas. $6^{\text {ta }}$ ed. Medellín, Corporación para investigaciones biológicas, 2003. pp.268-74.

Salerno, C.; Pascale, M.; Contaldo, M.; Esposito, V.; Busciolano, M.; Milillo, L.; et al. Candida-associated denture stomatitis. Med. Oral Patol. Oral Cir. Bucal., 16(2):139-43, 2011

Samaranayake, L. P.; Keung Leung, W. \& Jin, L. Oral mucosal fungal infections. Periodontol. 2000, 49:39-59, 2009

Shimizu, C.; Kuriyama, T.; Williams, D. W.; Karasawa, T.; Inoue, K.; Nakagawa, K.; et al. Association of oral yeast carriage with specific host factors and altered mouth sensation. Oral Surg. Oral Med. Oral Pathol. Oral Radiol. Endod., 105(4):445-51, 2008.

Williams, D. W.; Kuriyama, T.; Silva, S.; Malic, S. \& Lewis, M. A. Candida biofilms and oral candidosis: treatment and prevention. Periodontology 2000, 55(1):250-65, 2011.
Dirección para Correspondencia:

Prof. Dra. Ximena Lee M. DDs MSc

Directora Oficina Educación Odontológica

Presidenta Comisión Innovación Curricular

Facultad Odontología

Universidad de Chile

Santiago

CHILE

Email: ximenalee@gmail.com

Recibido : 04-10-2012

Aceptado: 23-04-2013 